\title{
Leistungsbezogenes Tarifsystem für die stationäre Psychiatrie
}

\author{
Beatrix Meyera, Bettina Holzer ${ }^{b}$ \\ a Leiterin Abteilung Tarife und Gesundheitsökonomie Spitalärzte \\ ${ }^{b}$ Dr. med., Abteilung Tarife und Gesundheitsökonomie Spitalärzte
}

\author{
Mit der Vorabversion des Tarifsystems TARPSY 0.2 werden verschiedene Varianten \\ aufgezeigt, die Tages- und Fallpauschalen kombinieren. Die FMH befürwortet eine \\ Tarifstruktur, die ausschliesslich auf leistungsbezogenen Tagespauschalen basiert. \\ Ein Fallpauschalenelement lehnt die FMH hingegen klar ab.
}

\begin{abstract}
Aktuell werden die Behandlungen in der stationären Psychiatrie mit Tagespauschalen vergütet, wobei deren Ausgestaltung je nach Kanton und Vertragspartner unterschiedlich ist. Der Gesetzgeber verlangt jedoch für die stationäre Psychiatrie ein national einheitliches, leistungsbezogenes Vergütungssystem. Dieses wird zurzeit unter dem Dach der SwissDRG AG erarbeitet. Ziel ist es, dass die neue Tarifstruktur einfach und praktikabel ist sowie möglichst wenige Fehlanreize aufweist. Die mit der Tarifstrukturentwicklung betraute Zürcher Hochschule für Angewandte Wissenschaften (ZHAW) legte inzwischen die Vorabversion TARPSY 0.2 vor. Dabei konzentrierte sich die ZHAW vorwiegend auf die Erwachsenenpsychiatrie. Für die Kinder- und Jugendpsychiatrie sind die Arbeiten aufgrund der ungenügenden Datenlage deutlich weniger weit fortgeschritten. Die Einführung von TARPSY ist für 2018 geplant.
\end{abstract}

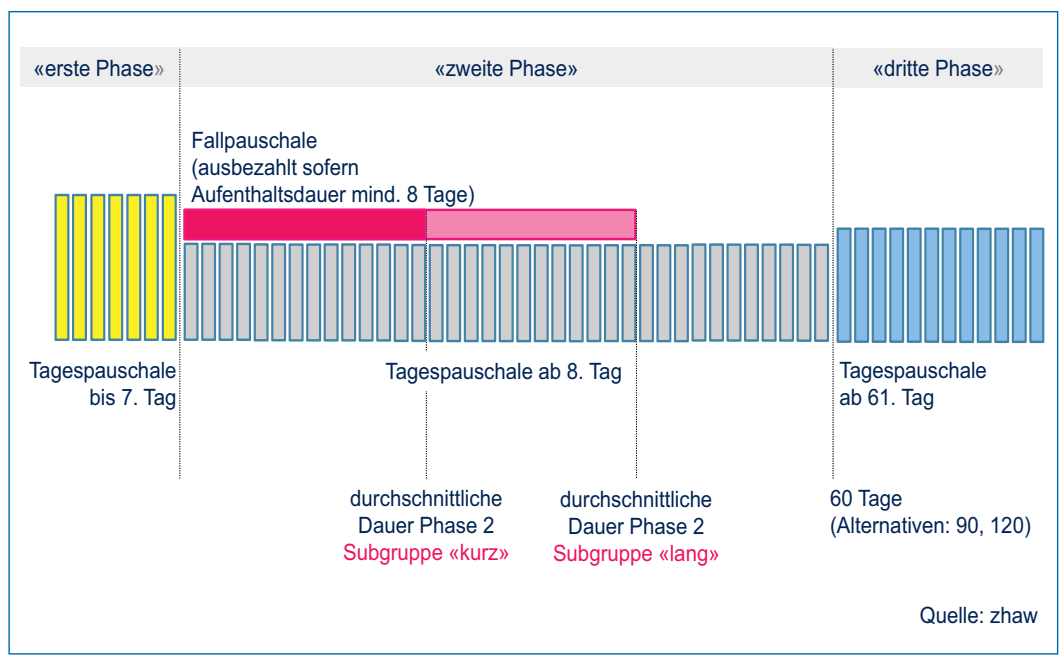

Abbildung 1: 3-Phasen-Tarifmodell für die stationäre Erwachsenenpsychiatrie.

\section{Drei-Phasen-Modell mit Tagespauschalen und Fallpauschalenelement}

Der stationäre Aufenthalt für die Erwachsenenpsychiatrie soll in drei Phasen aufgeteilt werden (Abb. 1). Die erste Phase dauert sieben Tage und wird mit Tagespauschalen vergütet. Ab dem achten Tag beginnt die zweite Phase, für welche eine Kombination von Tagesund Fallpauschalen vorgesehen ist. Für Langzeitpatienten folgt die dritte Phase wiederum mit Tagespauschalen. Es wird datengestützt geprüft, ob diese $a b$ dem 61., 91., oder 121. Tag beginnen soll.

Die psychiatrischen stationären Aufenthalte werden anhand der Hauptdiagnose sowie der Leistungsintensität in möglichst kostenhomogene psychiatrische Kostengruppen (PCG - psychiatric cost groups) unterteilt. Die Zuordnung zu einer der neun [1] psychiatrischen Hauptdiagnosegruppen erfolgt mit ICD 10 GM [2]. Für die Messung der Leistungsintensität schlägt die ZHAW für die Erwachsenenpsychiatrie HoNOS [3] vor.

Bei den Tagespauschalen in der Phase 1 und 2 wird innerhalb jeder psychiatrischen Diagnosegruppe anhand von HoNOS zwischen der Regel- und der Intensivbehandlung unterschieden. Bei einer Fallpauschale in Phase 2 werden zusätzlich mittels HoNOS Patientengruppen mit einer höheren bzw. tieferen prognostizierten Aufenthaltsdauer gebildet.

In Phase 1 und 2 ergeben sich somit je 18 PCG für die Tagespauschale [4]. Für die Fallpauschale in Phase 2 kommen 36 PCG hinzu [5]. Die Phase 3 berücksichtigt keine Leistungsintensität mehr und bildet nur drei PCG. Anhand der entsprechenden Kostengewichte pro PCG, der Aufenthaltstage sowie des von den Tarifpartnern verhandelten Basistarifs wird der Tarif für den stationären Aufenthalt berechnet [6]. 


\section{Fallpauschalen in der Psychiatrie ungeeignet}

Für die Phase 2 hat die ZHAW verschiedene Modellvarianten vorgestellt mit jeweils unterschiedlich hohen Fallpauschalenanteilen von 0\%, 25\%, 50\%, 75\% und $100 \%$. Dabei ist die Prognosegüte [7] umso besser, je geringer der Fallpauschalenanteil ist; am besten schneidet die Variante mit 0\% Fallpauschale bzw. 100\% Tagespauschale ab. Die ZHAW verweist darauf, dass durch einen Fallpauschalenanteil ein Anreiz entsteht, die Aufenthaltsdauer zu verkürzen. Die Höhe des Fallpauschalenanteils kann nicht datengestützt festgelegt werden, sondern ist durch die Partner der SwissDRG AG zu definieren.

Durch die Ausschüttung eines Fallpauschalenanteils nach Abschluss der 1. Phase würden sich Erlössprünge ergeben. Die SwissDRG AG möchte deshalb prüfen, wie Elemente des Modells TARPSY mit denjenigen des Modells SwissDRG kombiniert werden können, um Erlössprünge zu vermeiden.

\section{FMH lehnt Fallpauschalenelement klar ab und befürwortet leistungsbezogene Tages- pauschalen.}

Die FMH unterstützt eine Tarifstruktur, die zu 100\% aus Tagespauschalen besteht. Die Varianten mit einem Fallpauschalenanteil lehnt die FMH hingegen klar ab. Dies insbesondere deshalb, weil die Aufenthaltsdauer bei gleicher Diagnose nicht nur vom Arzt bzw. von der psychiatrischen Einrichtung abhängt, sondern auch vom Patienten und seinem sozialen Umfeld. Zudem sind Aufenthalte der fürsorgerischen Unterbringung (FU) oft nicht steuerbar. Die FU wird kantonal unterschiedlich gehandhabt, und so ist auch ihr Anteil je nach Kanton und Klinik verschieden hoch. Im Durchschnitt machen die Zwangseinweisungen in der Schweiz rund einen Viertel aller Einweisungen aus [8]. Mit dem Fallpauschalenelement befürchtet die FMH ausserdem, dass ein ökonomischer Druck für eine medizinisch nicht gerechtfertigte, zu frühe Entlassung der Patienten entsteht und entsprechend mehr Wiedereintritte erfolgen.

\section{Alternativen zu HoNOS werden geprüft}

Aus Sicht der FMH eignet sich HoNOS nicht, um die Leistungsintensität in der Erwachsenenpsychiatrie zu ermitteln. HoNOS wurde bisher für die Qualitätsmessung verwendet und getestet, nicht jedoch für die Tarifstrukturentwicklung. Zudem ist HoNOS nicht manipulationsresistent. Der BSCL [9] als Kontrollinstru- ment erwies sich gemäss der ZHAW als ungeeignet und wird nicht weiter verwendet; eine Alternative liegt jedoch nicht vor. Die Ärzteschaft befürchtet schliesslich einen höheren administrativen Aufwand, da die Erhebung von HoNOS für TARPSY aufwendiger ist als diejenige für den ANQ [10]. Die gleiche Problematik besteht mit HoNOSCA [11], welches die Leistungsintensität in der Kinder- und Jugendpsychiatrie abbilden soll. Die FMH forderte deshalb, dass andere Unterscheidungsmerkmale zur Ermittlung der Leistungsintensität wie etwa die fürsorgerische Unterbringung (FU) weiter geprüft werden. Inzwischen hat die ZHAW angekündigt, für TARPSY 0.3 abgesehen von HoNOS alternative Variablen wie die FU oder das Alter zu prüfen. Positiv ist zudem, dass die Forderung der FMH aus dem Positionspapier [12] zu TARPSY aufgenommen wurde, das multiaxiale Klassifikationsschema (MAS) als Unterscheidungsmerkmal für Kinder und Jugendliche zu evaluieren.

\section{Bessere Datenqualität ist entscheidend}

Grundlage für die Entwicklung von TARPSY 0.2 in der Erwachsenenpsychiatrie sind die Daten aus dem Jahr 2013 von 17 psychiatrischen Kliniken. Insgesamt konnte mangels Datenqualität nur ein Drittel der gelieferten Fälle genutzt werden, was knapp einem Fünftel der jährlich stationär behandelten Fälle entspricht. Um die Datenqualität bezüglich der FU zu verbessern, ist eine eindeutige Abgrenzung der statistischen Variablen «Freiwilligkeit» und «Fürsorgerischer Freiheitsentzug» (die frühere Bezeichnung der FU) notwendig, da die Erfassung dieser Variablen aktuell sehr uneinheitlich gehandhabt wird. In der Kinder- und Jugendpsychiatrie lieferten nur vier psychiatrische Kliniken Daten, zwei davon haben MAS erfasst. Für die Weiterentwicklung von TARPSY ist es daher wichtig, dass in Zukunft alle psychiatrischen Kliniken Daten in guter Qualität zur Verfügung stellen.

\section{Einbezug von Tages- und Nachtkliniken zentral}

Bedauerlicherweise wurden die Tages- und Nachtkliniken in TARPSY 0.2 nicht berücksichtigt. Aus Sicht der FMH sind diese jedoch in das Tarifsystem TARPSY einzubeziehen. Denn durch die aktuelle Untervergütung der Tages- und Nachtkliniken besteht der Anreiz, Patienten medizinisch unbegründet bzw. nur aus finanziellen Überlegungen stationär $\mathrm{zu}$ behandeln. Dies widerspricht dem Patientenwohl und verteuert das Gesundheitswesen unnötig. Es ist wichtig, dass die Patienten die für sie jeweils optimale Behandlung er- 
halten, unabhängig davon, ob diese stationär, in einer Tages- bzw. Nachtklinik, aufsuchend oder ambulant erbracht wird.

\section{Übergangsregelung und Begleitforschung notwendig}

Da es sich bei TARPSY um eine komplett neue Tarifstruktur handelt, ist aus Sicht der FMH eine mehrjährige Übergangsregelung erforderlich, um negative finanzielle Auswirkungen in der Einführungsphase zu vermeiden. Zudem ist bereits vor der TARPSY-Einführung eine Begleitforschung aufzugleisen. Nur durch

\section{In der Psychiatrie hängt die Behandlungsdauer stark vom sozialen Umfeld ab.}

eine frühzeitige Begleitforschung können mögliche Fehlentwicklungen der neuen Tarifstruktur rechtzeitig erkannt und entsprechende Gegenmassnahmen eingeleitet werden.

\section{Fazit und Ausblick}

Die FMH empfiehlt, das Tarifsystem TARPSY ausschliesslich auf leistungsbezogenen Tagespauschalen abzustützen und auf Fallpauschalen gänzlich zu verzichten. HoNOS und HoNOSCA sind nicht manipulationsresistent und aus Sicht der FMH damit ungeeignet, um die Leistungsintensität zu ermitteln. Deshalb begrüsst es die FMH ausdrücklich, dass die ZHAW für TARPSY 0.3 Alternativen prüft: beispielsweise die fürsorgerische Unterbringung in der Erwachsenenpsychiatrie und das multiaxiale Klassifikationsschema in der Kinderund Jugendpsychiatrie. Für die weitere Tarifstrukturentwicklung ist es wichtig, dass die Kliniken Daten in guter Qualität zur Verfügung stellen. Die FMH steht zusammen mit ihren Fachgesellschaften weiterhin aktiv für die Erarbeitung einer leistungsbezogenen Tarifstruktur zur Verfügung.

1 Geprüft wurde eine zusätzliche zehnte Hauptdiagnosegruppe für nicht-psychiatrische Hauptdiagnosen.

2 ICD 10 GM: Internationale statistische Klassifikation der Krankheiten und verwandter Gesundheitsprobleme. 10. Revision, German Modification, Grundlage der Diagnoseverschlüsselung in der med. Statistik der Schweiz.

3 HoNOS: Health of the Nation Outcome Scales, Fremdbewertungsinstrument.

4 Bei Verwendung der zusätzlichen zehnten Hauptdiagnosegruppe für nicht-psychiatrische Hauptdiagnosen kommt hier eine zusätzliche PCG dazu.

5 Bei Verwendung der zusätzlichen zehnten Hauptdiagnosegruppe für nicht-psychiatrische Hauptdiagnosen kommt hier eine zusätzliche PCG dazu.

6 Der Tarif in CHF wird dabei im Detail wie folgt berechnet: Basistarif in CHF $\times\left[\left(\right.\right.$ Tagespauschale $_{1} \times$ Aufenthaltsdauer $\left._{1}\right)+$ Fallpau- $^{-}$ schale $+\left(\right.$ Tagespauschale $_{2} \times$ Aufenthaltsdauer $\left.\left._{2}\right)\right]+\left(\right.$ Tagespauschale $_{3}$ $\times$ Aufenthaltsdauer $\left._{3}\right)$ ].

7 Die Prognosegüte der Modelle wurde ermittelt durch die Berechnung der durchschnittlichen Abweichung der Kosten eines Falls von den tatsächlichen Kosten.

8 Auswertung 2011 des Psychiatrie-Zusatzdatensatzes 2009 durch Obsan, vgl. Gassmann, Jürg, 2011. Wirksamkeit des Rechtsschutzes bei psychiatrischen Zwangseinweisungen in der Schweiz. Studie erstellt im Auftrag des Bundesamtes für Gesundheit. www.bag.admin.ch

9 BSCL: Brief Symptom Checklist, Selbstbewertungsinstrument.

10 ANQ: Nationaler Verein für Qualitätsentwicklung in Spitälern und Kliniken.

11 HoNOSCA: Health of the Nation Outcome Scales for Children and Adolescents.

12 Positionspapier der FMH zum Tarifsystem Psychiatrie (TARPSY), 2013, www.fmh.ch $\rightarrow$ Stationäre Tarife $\rightarrow$ Positionen $\rightarrow$ Positionspapiere. 